\title{
The Observation of Humoral Responses after Influenza Vaccination in Patients with Rheumatoid Arthritis Treated with Japanese Oriental (Kampo) Medicine: An Observational Study
}

\author{
Toshiaki Kogure, ${ }^{1}$ Naoyuki Harada, ${ }^{1}$ Yuko Oku, ${ }^{2}$ Takeshi Tatsumi, ${ }^{1}$ and Atsushi Niizawa ${ }^{3}$ \\ ${ }^{1}$ Department of Japanese Oriental Medicine, Gunma Central and General Hospital, Maebashi Gunma 371-0025, Japan \\ ${ }^{2}$ Department of Internal Medicine, Gunma Central and General Hospital, Maebashi Gunma 371-0025, Japan \\ ${ }^{3}$ Department of Japanese Oriental Medicine, Kobe Century Memorial Hospital, Japan
}

Correspondence should be addressed to Toshiaki Kogure, gch-kogure@kl.wind.ne.jp

Received 2 December 2011; Accepted 1 February 2012

Academic Editor: Vincenzo De Feo

Copyright (C) 2012 Toshiaki Kogure et al. This is an open access article distributed under the Creative Commons Attribution License, which permits unrestricted use, distribution, and reproduction in any medium, provided the original work is properly cited.

\begin{abstract}
Objective. The efficacy of influenza vaccination in patients treated with Japanese Oriental (Kampo) Medicine is unknown. The objectives of this study were to observe the efficacy of influenza vaccination in RA patients treated with Kampo. Methods. Trivalent influenza subunit vaccine was administered to 45 RA patients who had received Kampo. They were divided into 2 groups: RA patients treated without MTX ("without MTX group") and treated with MTX ("with MTX group"). Antibody titers were measured before and 4 weeks after vaccination using hemagglutination inhibition assay. Results. Geometric mean titers (GMTs) of antiinfluenza antibodies significantly increased for all influenza strains. Response to the influenza vaccination in RA patients treated with Kampo was not lower than that of healthy subjects and the response in the "with MTX group" had a tendency to be higher than that in RA patients treated with MTX in the previous study. There was no significant difference in the GMT after 4 weeks between the "with MTX group" and the "without MTX group." A decreased efficacy in both seroprotection and seroconversion was not found in the "with MTX group." Conclusion. These observations may open the way for further clinical trials to establish the efficacy for the influenza vaccination in RA patients treated with Kampo.
\end{abstract}

\section{Introduction}

Rheumatoid arthritis (RA) is a systemic autoimmune disease that is associated with immunologic changes in $\mathrm{T}$ cells and $\mathrm{B}$ cells. In patients with RA, an impaired ability to react to antigens and an increased peripheral blood CD4/CD8 ratio has been observed in $\mathrm{T}$ cells $[1,2]$. The presence of soluble interleukin-2 (IL-2) receptors in serum has showed $\mathrm{T}$ cell activation $[2,3]$. Furthermore, T cell receptor rearrangement excision circles measured from $\mathrm{T}$ cells from RA patients were substantially lower than those in healthy controls, because the $\mathrm{T}$ cell receptor repertoire has been oligoclonal, which suggests on antigen selection and restriction of the repertoire [4]. There is also a decline in the thymic output of $\mathrm{T}$ cells. This premature aging of $\mathrm{T}$ cells in RA may have very severe effects on vaccine responses, which are well known to decrease with aging [5]. Additionally, the function of regula- tory $\mathrm{T}$ cells (CD4+, CD25+) may be abnormal in active RA patients, with a lack of suppression of CD4+ or CD8+ T cells [6].

The multiple immunologic effects of the disease process may in part explain why patients with RA are considered immunocompromised and at increased risk of infection [7]. Therefore, although the exact prevalence, morbidity, and mortality of influenza in patients with RA are unknown, a yearly influenza vaccination is recommended [8]. The influenza vaccination is safe and results in protective levels of antiinfluenza antibodies in most RA patients, even when they are treated with prednisone, disease-modifying antirheumatic drugs (DMARDs), or tumor necrosis factor-blocking agents $[9,10]$.

In Japan, Japanese traditional herbal (Kampo) Medicine, which is covered by national health insurance, is often 
prescribed in the primary care field and is also applied as an alternative treatment for serious diseases such as RA. Since ancient times, many kinds of Kampo formulae have been used traditionally and are found to be clinically effective for RA treatment. These formulae usually contain components from several medicinal plants that are thought to exert anti-inflammation and immune-regulator effects and are effective for treating RA [11-13]. We have demonstrated that kampo formula possessed antirheumatic effects in vitro and in vivo $[14,15]$. Furthermore, we have observed that the administration of kampo formula partially suppressed $\mathrm{T}$ cell activation in collagen induced arthritis (CIA) mice [16]. However, the effectiveness of the influenza vaccination in RA patients treated with Kampo remedy is still not known. The purpose of this study is to investigate the response to the influenza vaccination in RA patients treated with Kampo remedy.

\section{Patients and Methods}

2.1. Patient's Profile. Patients who visited our department in 2010-2011 had to fulfill the American College of Rheumatology 1987 revised criteria for the classification of RA and were selected in a random sampling method. All patients had been treated with Kampo formulae, which were often administered to the patients with RA.

2.2. Study Design. An observational study design was utilized in this study. Forty-five patients were entered into this design. Patients received the influenza vaccine intramuscularly from October 2010 until January 2011. Immediately before and 4 weeks after vaccination, blood was drawn for the measurement of C-reactive protein levels (CRP), erythrocyte sedimentation rate (ESR), and anti-influenza antibodies. The Disease Activity Score in 28 joints (DAS28) [17] was recorded before and 4 weeks after vaccination. Information on previous influenza vaccinations was obtained from all participants, and adverse effects occurring in the first 7 days postvaccination were recorded. This study was approved by the Ethics Committee of Gunma Central \& General Hospital in Aug 2010.

2.3. Vaccine. We used a trivalent influenza subunit vaccine (2010-2011; Daiichi-Sankyo co.ltd Tokyo Japan) containing purified hemagglutinin and neuramidase of the following strains: A/California/7/2009 (H1N1)-like strain (A/H1N1 strain), A/Victoria/210/2009 (H3N2)-like strain (A/H3N2 strain), and B/Brisbane/60/2008-like strain (B strain).

2.4. Hemagglutination Inhibition Assay (HIA). The HIA was used for the detection of anti-influenza antibodies. HIAs were performed with guinea pig erythrocytes in accordance with standard procedures [18]. The following parameters for efficacy of the vaccination based on the anti-influenza antibody response were evaluated: geometric mean titer (GMT), fold increase in titer, 4 -fold titer rise resulting in a postvaccination level of 40 (seroconversion), and titer rise to $40 \geq$ (seroprotection). HIA titers 40 are generally considered to be protective in healthy adults [19].

\section{Results}

3.1. Patient Characteristics. Forty-five RA patients were administered Kampo treatment. They were divided into 2 groups as follows: 16 RA patients treated without MTX (without MTX group) and 23 RA patients treated with MTX (with MTX group). Patients treated with tacrolimus (TAC) or biologics were excluded from the patients in the without MTX group, and patients treated with biologics were excluded from the patients in both the with MTX and without MTX group. Their characteristics were shown in Table 1.

3.2. The Response to the Influenza Vaccination. Each GMT after 4 weeks vaccination was $78.8 \pm 119.7,35.7 \pm 33.6$, and $27.3 \pm 27.3$ in $\mathrm{A} / \mathrm{H} 1 \mathrm{~N} 1, \mathrm{~A} / \mathrm{H} 3 \mathrm{~N} 2$, and $\mathrm{B}$ strain, respectively (Table 2). Response to the influenza vaccination in RA patients treated with Kampo formulae was not lower than that of healthy subjects in previous studies [20,21]. There was no significant difference in the GMT after 4 weeks between the "with MTX group" and the "without MTX group." The GMT in the with MTX group was higher than in the without MTX group (Table 2). The response in the with MTX group had a tendency to be higher than that in RA patients treated with MTX in the previous study [21]. Furthermore, we calculated the fold increase as well as the GMT. The mean fold increase in each group was as follows: 6.5, 2.6, and 2.1, respectively (Table 2). The fold increase in the with MTX group also had a tendency to be higher than in the without MTX group, although this was not significant.

3.3. Seroprotection and Seroconversion. After 4 weeks vaccination, the percentage of patients who possessed the $40 \geq$ titer in $\mathrm{A} / \mathrm{H} 1 \mathrm{~N} 1$ was $53.3,50.0$, and $65.2 \%$ in total RA patients, without MTX group and with MTX group, respectively (Figure 1). There was no significant difference between the with MTX and the without MTX groups and a decreased efficacy in seroprotection was not found in the with MTX group. In $\mathrm{A} / \mathrm{H} 3 \mathrm{~N} 2$, the percentage of patients who possessed the $40 \geq$ titer was $46.7,50.0$, and $52.2 \%$, and in the B strain, 28.9, 25.0, and $39.1 \%$ in total RA patients, without MTX group, and with MTX group, respectively. The seroprotection effect observed in the with MTX group had a tendency to be higher than results in the previous study [21]. In seroconversion, the percentage of patients who possessed 40 $\geq$ titer induced by 4 -fold increase was $40.0,35.6$, and $15.6 \%$, respectively $(\mathrm{A} / \mathrm{H} 1 \mathrm{~N} 1, \mathrm{~A} / \mathrm{H} 3 \mathrm{~N} 2$, and B Strain). There was no significant difference between the with MTX and the without MTX groups also in seroconversion (data not shown).

3.4. The Influence of Influenza Vaccination upon RA Disease Activity. The DAS28 did not change after vaccination. There was no adverse reaction by influenza vaccination.

\section{Discussion}

Kampo medicine, which is covered by national health insurance in Japan, is often prescribed in the primary care field, 
TABLE 1: Characteristics at baseline of RA patients in this study.

\begin{tabular}{lccc}
\hline & Total & Without MTX group* & With MTX group** \\
\hline Age, mean \pm SD years & $56.2 \pm 13.5$ & $58.6 \pm 10.5$ & $54.1 \pm 12.6$ \\
No. (\%) female/No. (\%) male & $42(93) / 3(7)$ & $15(94) / 1(6)$ & $22(92) / 2(8)$ \\
Duration of RA mean \pm SD years & $12.2 \pm 14.1$ & $13.5 \pm 15.6$ & $10.9 \pm 11.6$ \\
MTX dosage, mean \pm mg/week & $5.1 \pm 3.8$ & 0 & $7.6 \pm 2.5$ \\
PSL dosage, mean \pm SD mg/day & $2.1 \pm 2.0$ & $1.6 \pm 1.5$ & $2.4 \pm 1.9$ \\
Taking DMARDs, No. & & & 0 \\
$\quad$ Bucillamine & 1 & 8 & 2 \\
$\quad$ Sulfasalazine & 11 & 0 & 4 \\
$\quad$ Tacllolimus & $4.2 \pm 1.1$ & $2.9 \pm 1.0$ & $3.3 \pm 1.4$ \\
DAS28 CRP & & & 0 \\
\hline
\end{tabular}

${ }^{*}$ Without MTX group: patients treated with classical DMARDs alone. Patients treated with tacllolimus were excluded. ${ }^{* *}$ with MTX group: patients treated with MTX, but not biologics.

TABLE 2: GMTs and fold increase in GMT for influenza A/H3N2, A/H1N1, and B strains in RA patients treated with Kampo formulae before and after administration of influenza vaccines.

\begin{tabular}{|c|c|c|c|}
\hline & Total & Without MTX group* & $\begin{array}{c}\text { With MTX } \\
\text { group** }\end{array}$ \\
\hline \multicolumn{4}{|l|}{ GMT, mean \pm SD } \\
\hline \multicolumn{4}{|l|}{ A/H1N1 strain } \\
\hline Baseline & $12.1 \pm 14.0$ & $11.0 \pm 12.1$ & $14.1 \pm 15.0$ \\
\hline 4 weeks later & $78.8 \pm 119.7$ & $39.6 \pm 39.3$ & $115.9 \pm 148.8$ \\
\hline \multicolumn{4}{|l|}{$\mathrm{A} / \mathrm{H} 3 \mathrm{~N} 2$ strain } \\
\hline Baseline & $13.5 \pm 13.9$ & $16.0 \pm 19.7$ & $11.7 \pm 10.2$ \\
\hline 4 weeks later & $35.7 \pm 33.6$ & $33.1 \pm 21.8$ & $39.1 \pm 40.2$ \\
\hline \multicolumn{4}{|l|}{ B strain } \\
\hline Baseline & $12.8 \pm 10.3$ & $13.9 \pm 9.2$ & $11.4 \pm 11.5$ \\
\hline 4 weeks later & $27.3 \pm 27.8$ & $22.8 \pm 19.2$ & $31.4 \pm 34.0$ \\
\hline \multicolumn{4}{|c|}{ Fold increase, mean (range) } \\
\hline A/H1N1 strain & $6.5(1$ to 64$)$ & $3.6(1$ to 16$)$ & $8.2(1$ to 64$)$ \\
\hline $\mathrm{A} / \mathrm{H} 3 \mathrm{~N} 2$ strain & $2.6(1$ to 16$)$ & $2.1(1$ to 8$)$ & $3.3(1$ to 16$)$ \\
\hline B strain & $2.1(1$ to 16$)$ & $1.6(1$ to 4$)$ & $2.7(1$ to 16$)$ \\
\hline
\end{tabular}

${ }^{*}$ Without MTX group: patients treated with classical DMARDs alone. Patients treated with tacllolimus were excluded. ${ }^{* *}$ with MTX group: patients treated with MTX, but not biologics.

and is also applied as an alternative remedy for RA. The efficacy for RA of Kampo medicines has been demonstrated by case or case series reports and several clinical trials. From these reports, the clinical effectiveness of Kampo therapy is almost similar to that of classical DMARDs, such as bucillamine $(\mathrm{Bc})$ and salazosulfapyridine (SASP). Additionally, several investigators have demonstrated the immunomodulatory effects of Kampo medicine in RA patients as well as an arthritis mouse model, such as CIA $[11,12,14]$. We have also reported that Kampo therapy resulted in a decrease in serum IL-6 levels, but not TNF- $\alpha$ levels, as well as the suppression of arthritis development, based on the observations of the CIA mouse model [15]. Furthermore, it has been reported that Kampo medicine is probably effective against infection. The efficacy of Kampo therapy on atypical mycobacterium pneumonia and aspiration bacterial pneumonia has been demonstrated $[22,23]$, and these effects may be caused by immune-regulator effects, but not direct antibacterial effects. On the other hand, RA patients are susceptible to both viral and bacterial infections. In Japanese RA patients, major causes of death included malignancies $(24.2 \%)$, respiratory involvement $(24.2 \%)$ including pneumonia (12.1\%) and interstitial lung disease (ILD) (11.1\%), cerebrovascular disease (8.0\%), and myocardial infarction (7.6\%) [24]. Infectious disease is one of the critical factors in the mortality of RA patients. Therefore, a yearly influenza vaccination is recommended by the Center for Disease Control and Prevention (CDC) [25, 26]. However, the immune response to the influenza vaccination has not been reported in RA patients treated with Kampo medicine. This is the first report demonstrating the titer of anti-influenza antibodies before and after influenza vaccination in RA patients administered Kampo formulae.

The response to the influenza vaccination in our population was almost similar to previous results in healthy subjects. Kampo therapy may be beneficial for RA patients from the clinical viewpoint of protection against influenza 


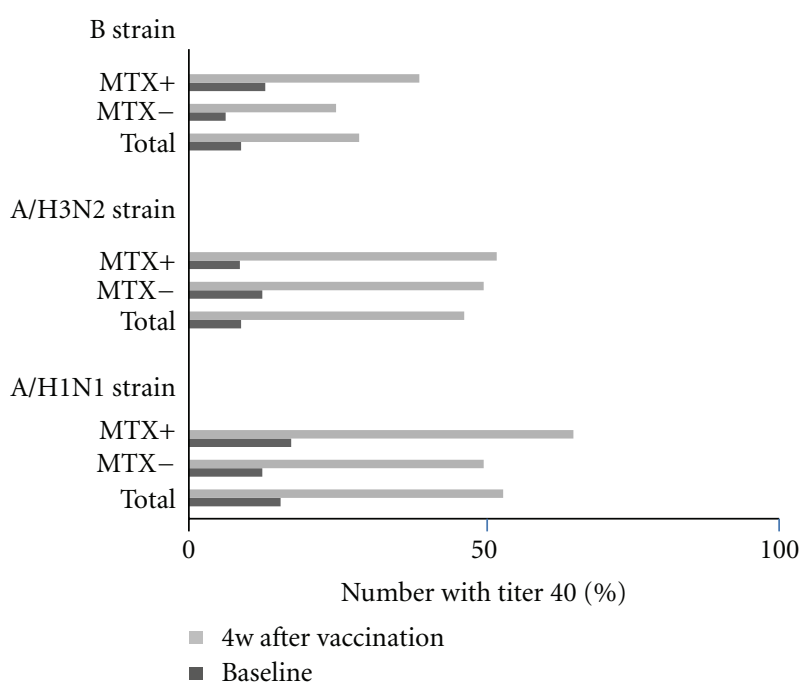

Figure 1: Percentage of patients with anti-influenza titers $\geq 40$, as determined by a hemagglutination inhibition assay for each strain after vaccination with a trivalent influenza subunit vaccine, in total RA patients, RA patients treated with MTX, and RA patients treated without MTX. Solid bars represent prevaccination titer $\geq 40$; open bars represent post vaccination titer $\geq 40$.

virus infection as well as suppression of RA disease activity. However, there are various opinions about the efficacy of the influenza vaccination in RA patients. Some reports demonstrate both no differences and significant differences in the response rate between treatment with and without MTX in RA patients [20, 27-29]. This discrepancy may be caused by the different endpoints when measuring the response to the influenza vaccination and different influenza virus roots. Therefore, our data should be limited in reference to the adjuvant effects of Kampo therapy. However, as the baseline titers in this study were less than previous studies, we consider Kampo therapy to be partially beneficial for RA patients in seroprotection and seroconversion. In addition, it has been reported that the response to vaccination was significantly less in patients treated with anti-TNF-a and antiCD20 antibody (rituximab) drugs than RA patients without biologics [21,29]. We have checked the titers of the 5 patients treated with biologics, and they were less than those of other RA patient groups (data not shown). Kampo therapy may not influence the response to the influenza vaccination in RA patients treated with biologics. To analyze this problem, further clinical observational studies will be required using a large number of patients.

The RA disease activity by DAS28 did not change after vaccination in our patients. It is generally thought that the vaccination does not influence the disease activity and the titer of the serological markers. A recent report demonstrates that influenza vaccination did not alter the percentage of healthy adults with positive autoantibodies [30].

We have reported several patients with MTX-resistant RA as being successfully treated with Kampo medicine; however, it is still not clear as to how Kampo medicine acts on arthritis in humans [31]. We previously demonstrated that Kam- po medicine suppressed polyclonal B cell activation, but not $\mathrm{T}$ cell activation, significantly in the CIA mouse model [14, 15]. Recently, it has been clarified that the development of arthritis in the CIA mouse contributed to the differentiation of IL-17 producing cells (Th17), dependent on IL- 6 and TGF- $\beta[32,33]$. In our previous study using CIA, Kampo medicine decreased the serum IL-6 levels, but not TNF- $\alpha$, suggesting that the suppression of Th17 cell activation by Kampo therapy probably improved the development of arthritis. Thus, we suggest that Kampo medicines do not influence the function of antigen presentation in dendrite cells or macrophages. Based on these findings, we suggest that Kampo therapies do not suppress the response to the influenza vaccination in RA patients. Besides, in innate immunity, we have demonstrated that Juzentaihoto enhanced the production of iNOS in macrophages [34] and the upregulation of NK receptor's expression (Killer-cell immunoglobulin-like receptors) in NK cells [35]. Additionally, the direct anti-influenza virus actions of cinnamon cortex and ephedrae herba (the main herbs composing kampo formulae) have been demonstrated, while these actions are not associated with the response to vaccination in RA patients treated with Kampo $[36,37]$.

In conclusion, we have demonstrated the changes in the titer of each anti-influenza antibody before and after vaccination in RA patients treated with Kampo formula. A low response to the vaccination was not observed compared with previous studies, and in the MTX-treated patients group, the response to vaccination was higher in our study than in previous reports. The present observations may open the way for further clinical trials to establish the efficacy for the influenza vaccination in RA patients treated with Kampo medicines.

\section{Acknowledgment}

This paper was supported by a Grant-in-Aid for Scientific Research from the Ministry of Health, Labor, and Welfare in Japan.

\section{References}

[1] J. Verwilghen, S. Vertessen, E. A. Stevens, J. Dequeker, and J. L. Ceuppens, "Depressed T-cell reactivity to recall antigens in rheumatoid arthritis," Journal of Clinical Immunology, vol. 10, no. 2, pp. 90-98, 1990.

[2] J. C. Beckham, D. S. Caldwell, B. L. Peterson et al., "Disease severity in rheumatoid arthritis: relationships of plasma tumor necrosis factor-alpha, soluble interleukin 2-receptor, soluble $\mathrm{CD} 4 / \mathrm{CD} 8$ ratio, neopterin, and fibrin $\mathrm{D}$-dimer to traditional severity and functional measures," Journal of Clinical Immunology, vol. 12, no. 5, pp. 353-361, 1992.

[3] T. Kogure, T. Itoh, Y. Shimada, T. Shintani, H. Ochiai, and K. Terasawa, "Detection of serum soluble markers of immune activation in rheumatoid arthritis," Mediators of Inflammation, vol. 5, no. 4, pp. 262-265, 1996.

[4] K. Koetz, E. Bryl, K. Spickschen, W. M. O’Fallon, J. J. Goronzy, and C. M. Weyand, "T cell homeostasis in patients with rheumatoid arthritis," Proceedings of the National Academy of Sciences of the United States of America, vol. 97, no. 16, pp. 9203 $9208,2000$. 
[5] T. M. Govaert, C. T. Thijs, N. Masurel, M. J. Sprenger, G. J. Dinant, and J. A. Knottnerus, "The efficacy of influenza vaccination in elderly individuals: a randomized double-blind placebo-controlled trial," Journal of the American Medical Association, vol. 272, no. 21, pp. 1661-1665, 1994.

[6] M. R. Ehrenstein, J. G. Evans, A. Singh et al., "Compromised function of regulatory $\mathrm{T}$ cells in rheumatoid arthritis and reversal by anti-TNF $\alpha$ therapy," Journal of Experimental Medicine, vol. 200, no. 3, pp. 277-285, 2004.

[7] F. Wolfe, D. M. Mitchell, J. T. Sibley et al., "The mortality of rheumatoid arthritis," Arthritis and Rheumatism, vol. 37, no. 4, pp. 481-494, 1994.

[8] Centers for Disease Control and Prevention, "Prevention of pneumococcal disease: recommendations of the advisory committee on immunization practices (ACIP)," Morbidity and Mortality Weekly Report, vol. 55, pp. 1-42, 2006.

[9] I. Fomin, D. Caspi, V. Levy et al., "Vaccination against influenza in rheumatoid arthritis: the effect of disease modifying drugs, including TNF $\alpha$ blockers," Annals of the Rheumatic Diseases, vol. 65, no. 2, pp. 191-194, 2006.

[10] A. Chalmers, D. Scheifele, C. Patterson et al., "Immunization of patients with rheumatoid arthritis against influenza: a study of vaccine safety and immunogenicity," Journal of Rheumatology, vol. 21, no. 7, pp. 1203-1206, 1994.

[11] D. M. Chang, W. Y. Chang, S. Y. Kuo, and M. L. Chang, "The effects of traditional antirheumatic herbal medicines on immune response cells," Journal of Rheumatology, vol. 24, no. 3, pp. 436-441, 1997.

[12] K. Asano, J. Matsuishi, Y. Yu, T. Kasahara, and T. Hisamitsu, "Suppressive effects of Tripterygium wilfordii Hook f., a traditional Chinese medicine, on collagen arthritis in mice," Immunopharmacology, vol. 39, no. 2, pp. 117-126, 1998.

[13] S. Kobayashi, H. Kobayashi, H. Matsuno, I. Kimura, and M. Kimura, "Inhibitory effects of anti-rheumatic drugs containing magnosalin, a compound from "Shin-I" (Flos magnoliae), on the proliferation of synovial cells in rheumatoid arthritis models," Immunopharmacology, vol. 39, no. 2, pp. 139-147, 1998.

[14] A. Niizawa, T. Kogure, L. X. Hai et al., "Clinical and immunomodulatory effects of Fun-boi, an herbal medicine, on collagen-induced arthritis in vivo," Clinical and Experimental Rheumatology, vol. 21, no. 1, pp. 57-62, 2003.

[15] L. X. Hai, T. Kogure, A. Niizawa et al., "Suppressive effect of hochu-ekki-to on collagen induced arthritis in DBA1J mice," Journal of Rheumatology, vol. 29, no. 8, pp. 1601-1608, 2002.

[16] T. Kogure, T. Tatsumi, A. Niizawa, H. Fujinaga, Y. Shimada, and K. Terasawa, "The population of CD40L-expressing cells was slightly but not significant decreased in lymphoid tissues of collagen induced arthritic mice treated with Hochu-EkkiTo," Yakugaku Zasshi, vol. 127, no. 3, pp. 547-550, 2007.

[17] M. L. Prevoo, M. A. van't Hof, H. H. Kuper, M. A. van Leeuwen, L. B. van de Putte, and P. L. van Riel, "Modified disease activity scores that include twenty-eight-joint counts: development and validation in a prospective longitudinal study of patients with rheumatoid arthritis," Arthritis and Rheumatism, vol. 38, no. 1, pp. 44-48, 1995.

[18] A. Holvast, A. Huckriede, J. Wilschut et al., "Safety and efficacy of influenza vaccination in systemic lupus erythematosus patients with quiescent disease," Annals of the Rheumatic Diseases, vol. 65, no. 7, pp. 913-918, 2006.

[19] J. C. de Jong, A. M. Palache, W. E. Beyer, G. F. Rimmelzwaan, A. C. Boon, and A. D. Osterhaus, "Haemagglutination-inhibiting antibody to influenza virus," Developments in Biologicals, vol. 115, pp. 63-73, 2003.
[20] I. Fomin, D. Caspi, V. Levy et al., "Vaccination against influenza in rheumatoid arthritis: the effect of disease modifying drugs, including TNF $\alpha$ blockers," Annals of the Rheumatic Diseases, vol. 65, no. 2, pp. 191-194, 2006.

[21] S. van Assen, A. Holvast, C. A. Benne et al., "Humoral responses after influenza vaccination are severely reduced in patients with rheumatoid arthritis treated with rituximab," Arthritis and Rheumatism, vol. 62, no. 1, pp. 75-81, 2010.

[22] T. Nogami, N. Sekiya, T. Mitsuma, and T. Yamaguchi, "A case of pulmonary Mycobacterium fortuitum infection successfully treated with Kampo treatments," Kekkaku, vol. 81, no. 8, pp. 525-529, 2006.

[23] N. Mantani, Y. Kasahara, T. Kamata et al., "Effect of Seihaito, a Kampo medicine, in relapsing aspiration pneumonia-an open-label pilot study," Phytomedicine, vol. 9, no. 3, pp. 195201, 2002.

[24] A. Nakajima, E. Inoue, E. Tanaka et al., "Mortality and cause of death in Japanese patients with rheumatoid arthritis based on a large observational cohort, IORRA," Scandinavian Journal of Rheumatology, vol. 39, no. 5, pp. 360-367, 2010.

[25] M. Bijl, N. Agmon-Levin, J. M. Dayer, E. Israeli, M. Gatto, and Y. Shoenfeld, "Vaccination of patients with auto-immune inflammatory rheumatic diseases requires careful benefit-risk assessment," Autoimmunity Reviews, In press.

[26] F. Conti, S. Rezai, and G. Valesini, "Vaccination and autoimmune rheumatic diseases," Autoimmunity Reviews, vol. 8, no. 2, pp. 124-128, 2008.

[27] L. Stojanovich, "Influenza vaccination of patients with systemic lupus erythematosus (SLE) and rheumatoid arthritis (RA)," Clinical and Developmental Immunology, vol. 13, no. 24, pp. 373-375, 2006.

[28] J. Sibilia and J. F. Maillefert, "Vaccination and rheumatoid arthritis," Annals of the Rheumatic Diseases, vol. 61, no. 7, pp. 575-576, 2002.

[29] S. Oren, M. Mandelboim, Y. Braun-Moscovici et al., "Vaccination against influenza in patients with rheumatoid arthritis: the effect of rituximab on the humoral response," Annals of the Rheumatic Diseases, vol. 67, no. 7, pp. 937-941, 2008.

[30] N. Toplak, T. Kveder, A. Trampus-Bakija, V. Subelj, S. Cucnik, and T. Avcin, "Autoimmune response following annual influenza vaccination in 92 apparently healthy adults," Autoimmunity Reviews, vol. 8, no. 2, pp. 134-138, 2008.

[31] T. Kogure, H. Sato, D. Kishi, T. Ito, and T. Tatsumi, "Serum levels of anti-cyclic citrullinated peptide antibodies are associated with a beneficial response to traditional herbal medicine (Kampo) in rheumatoid arthritis," Rheumatology International, vol. 29, no. 12, pp. 1441-1447, 2009.

[32] C. A. Murphy, C. L. Langrish, Y. Chen et al., "Divergent pro- and antiinflammatory roles for IL-23 and IL-12 in joint autoimmune inflammation," Journal of Experimental Medicine, vol. 198, no. 12, pp. 1951-1957, 2003.

[33] S. Nakae, A. Nambu, K. Sudo, and Y. Iwakura, "Suppression of immune induction of collagen-induced arthritis in IL-17-deficient mice," Journal of Immunology, vol. 171, no. 11, pp. 61736177, 2003.

[34] H. Kawamata, H. Ochiai, N. Manlani, and K. Terasawa, "Enhanced expression of inducible nitric oxide synthase by JuzenTaiho-To in LPS-activated RAW264.7 Cells, a murine macrophage cell line," American Journal of Chinese Medicine, vol. 28, no. 2, pp. 217-226, 2000.

[35] T. Kogure, A. Niizawa, L. X. Hai et al., "Effect of interleukin 2 on killer cell inhibitory receptors in patients with rheumatoid arthritis," Annals of the Rheumatic Diseases, vol. 60, no. 2, pp. 166-169, 2001. 
[36] K. Hayashi, N. Imanishi, Y. Kashiwayama et al., "Inhibitory effect of cinnamaldehyde, derived from Cinnamomi cortex, on the growth of influenza A/PR/8 virus in vitro and in vivo," Antiviral Research, vol. 74, no. 1, pp. 1-8, 2007.

[37] N. Mantani, N. Imanishi, H. Kawamata, K. Terasawa, and H. Ochiai, "Inhibitory effect of $(+)$-catechin on the growth of influenza A/PR/8 virus in MDCK cells," Planta Medica, vol. 67, no. 3, pp. 240-243, 2001. 


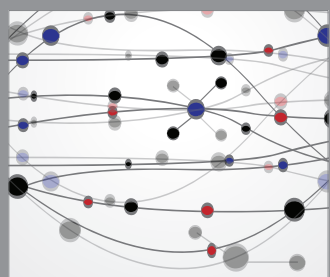

The Scientific World Journal
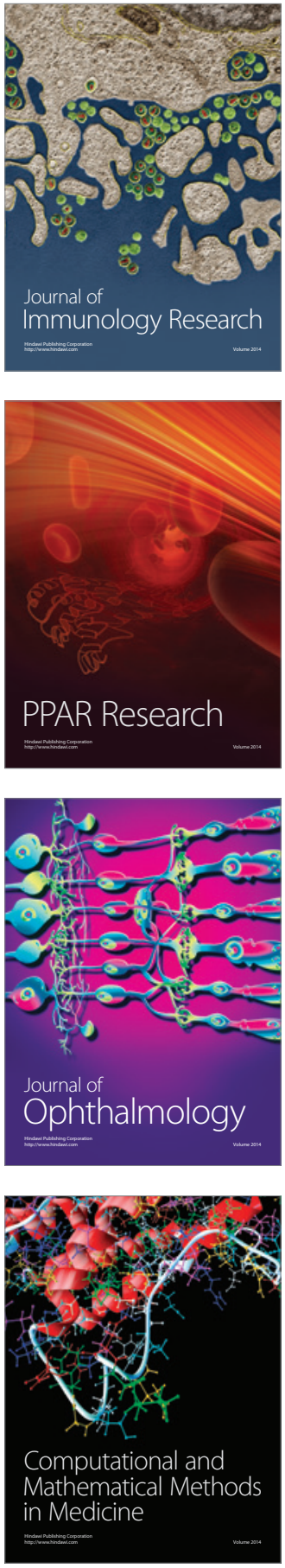

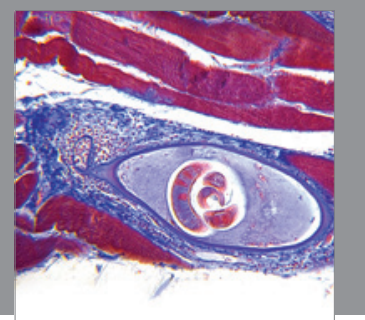

Gastroenterology

Research and Practice
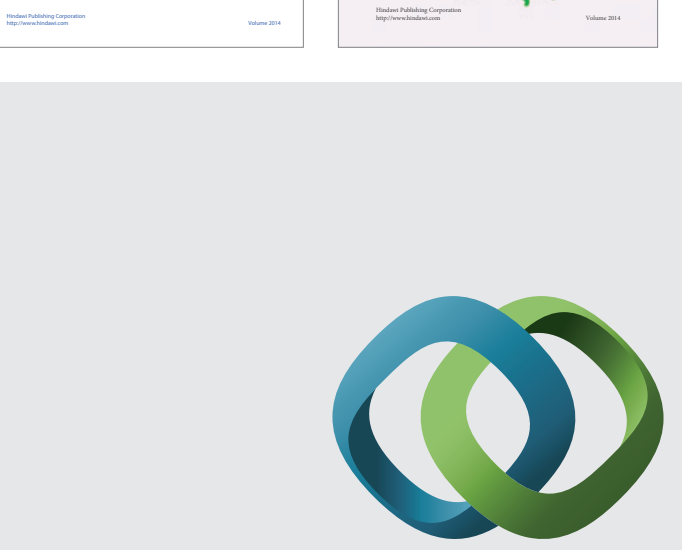

\section{Hindawi}

Submit your manuscripts at

http://www.hindawi.com
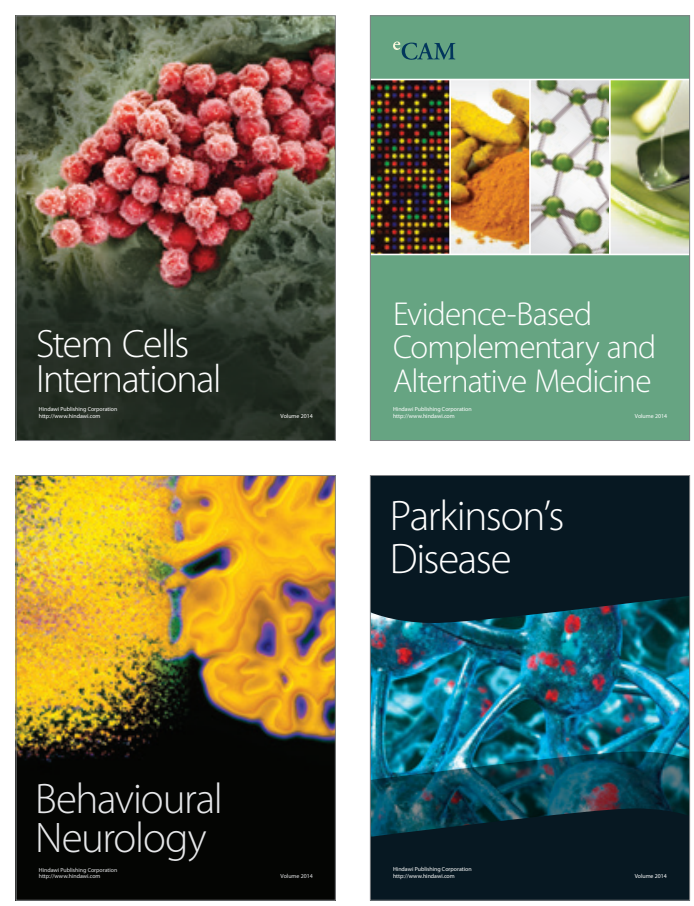

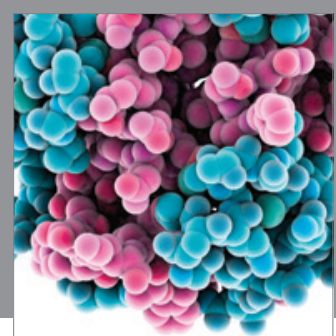

Journal of
Diabetes Research

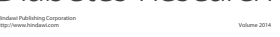

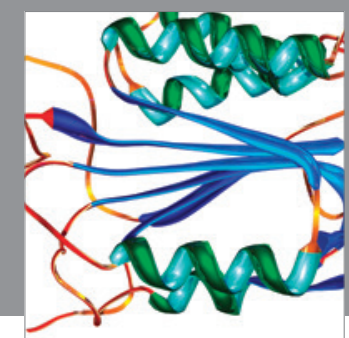

Disease Markers
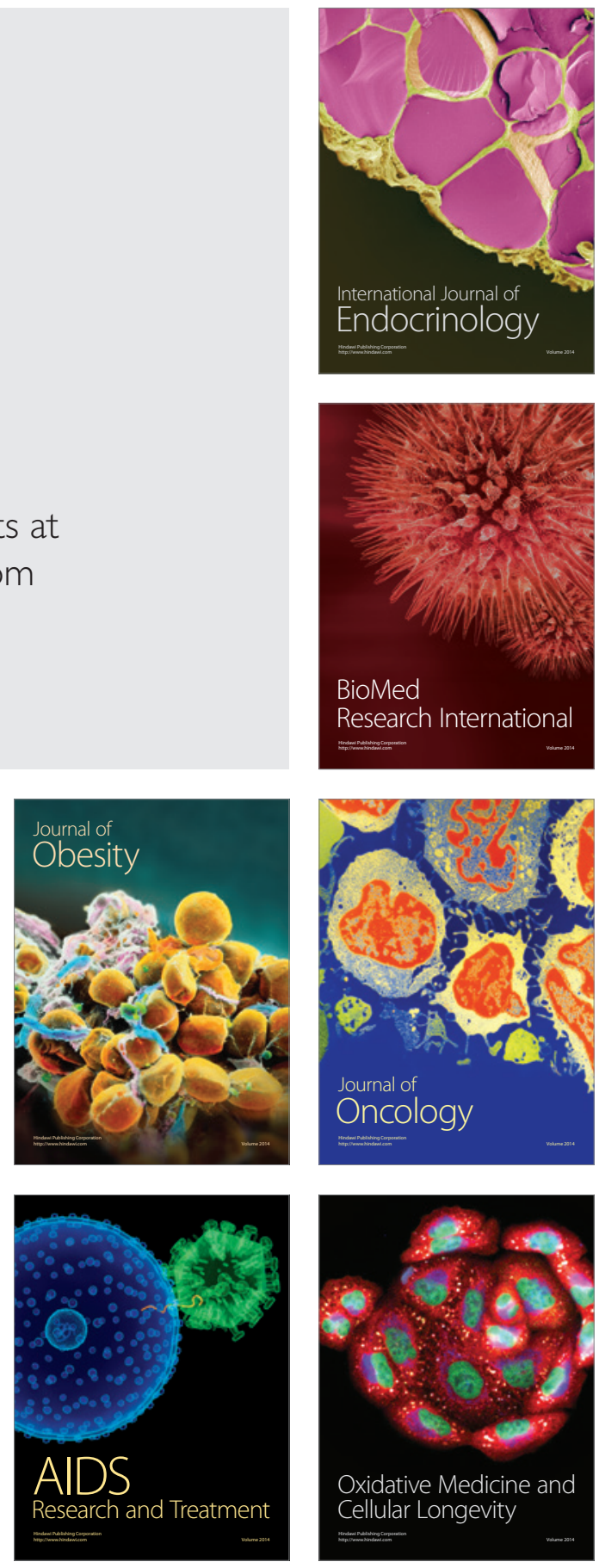